\title{
The number of dysplastic and common nevi in patients with malignant melanoma
}

\section{Fatma Pelin Cengiz, Kadriye Sallahoglu, Nazan Emiroglu, Nahide Onsun}

\author{
Department of Dermatology, Bezmialem Vakif University, Istanbul, Turkey \\ Corresponding author: Dr. Fatma Pelin Cengiz, E-mail: fpelinozgen@hotmail.com
}

\begin{abstract}
Background: There is no data about melanocytic lesions in Turkish patients with melanoma. We aimed to investigate number of melanocytic nevi in melanoma patients compared with control subjects. Methods: We performed a retrospective study in 78 melanoma patients and 78 control subjects. Clinical data were recorded for all participants. Results: As compared with control subjects, patients had more common nevi than control subjects on the legs. The patients and control subjects had similar number of overall and dysplastic nevi. Conclusions: Furthermore, the number of nevi is not a risk factor for our patients, and patients with few or no moles may develop MM, all patients need attention.
\end{abstract}

Key words: Dysplastic; Nevi; Melanoma

\section{INTRODUCTION}

The dysplastic nevus was first described in the 1970s in families prone to develop melanoma. It was believed that these lesions had a higher risk of transformation to melanoma than common nevi $[1,2]$. In 1992, Consensus Conference at the National Institutes of Health (NIH) described the criteria for dysplastic nevus [3]. Some of the authors suggest that nevus known as dysplastic nevus are actually congenital nevus. It is established that patients with melanoma have more Dysplastic nevi and common nevi than patients without melanoma [4-7]. We aimed to determine the number of nevi in melanoma patients.

\section{MATERIALS AND METHODS}

We performed a retrospective study on patients with malignant melanoma, who were recruited from those consecutively attending the outpatient clinics of department of dermatology at Bezmialem Vakif University, during January 2005 through December 2016. Seventy eight melanoma patients and age-gender matched 78 control subjects, were enrolled in the study. The study was approved by the local ethics committee. Informed consent was obtained from all participants, and the study was conducted according to the Declaration of Helsinki Principles. Patients and controls fulfilled the following criteria: (1) no positive family history of melanoma; (2) not referred for another skin cancer; (3) no previous history of phototherapy or any immunosupression therapy and; (4) no previous history of malignancy (5) with a Fitzpatrick skin type 4 or 5.

For all participants, melanocytic nevi which have atypical dermoscopic and clinical features at first visit (from $5-15 \mathrm{~mm}$ in diameter, flatness, indistinct borders, and color irregularity) followed up for 5 years and more without melanoma development, and common nevi (size $>2 \mathrm{~mm}$, sharp border, homogeneous light to dark brown color) were counted on all body sites (including palms, soles, scalp, and genital areas) and recorded using categories $(0 ; 0-10 ; 11-50 ; 51-100 ;>100)$.

Dermoscopic images of all the lesions were acquired at a $30 \mathrm{X}$ magnification and were stored in a digital imaging system (Fotofinder). All digital images were examined by experted dermatologist in a blinded fashion and were evaluated for global and local features.

All melanomas included in the analysis were; $3(1.9 \%)$ patients with lentigo maligna melanoma, 7 (4.5\%)

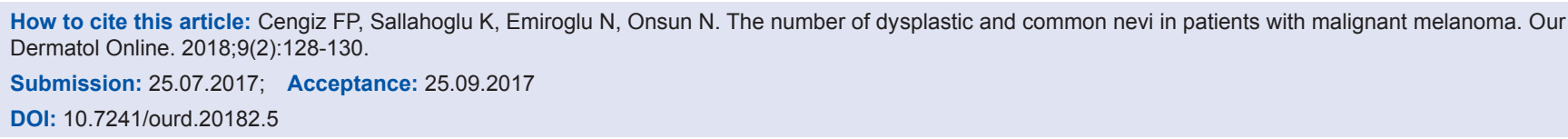


with acral lentiginous melanoma, 40 (25.6\%) with superficial spreading melanoma, $13(8.3 \%)$ with nodular melanoma, $3(1.9 \%)$ patients with spitzoid melanoma, $8(5.1 \%)$ patients with in situ melanoma and $4(2.6 \%)$ with unknown origin.

\section{Statistical Analysis}

Continuous variables were compared using Student $t$ test and Mann-Whitney-U test. For categorical variables, different groups were compared using chi-square tests. All statistical tests were 2-tailed and considered significant for $\mathrm{P}$ values of 0.05 or less. The analysis was carried out using SPSS.

\section{RESULTS}

\section{Characteristics of the Study Population}

A total of 156 participants, 78 melanoma patients $[31$ male and 47 female; median age: 55 years (range 16-89 years)] and 78 control subjects [31 male and 47 female; median age: 50 years (range 16-74 years)] were included in the study (Table 1).

We found a wide variation in the total number of nevi both in melanoma and control groups (from 0 to 226). Melanoma patients had more nevi than control subjects, both in the overall nevus count and in the number of atypical nevi, but both of them were not statistically significant (P:0.260 and P:0.382, respectively) (Table 1).

In detail, 1 of $78(1.28 \%)$ patients versus 1 of $78(1.28 \%)$ control subjects had no nevi, 26 of $78(33.3 \%)$ patients versus 13 of $78(16.7 \%)$ control subjects had 1 to 10 nevi, 30 of 78 (38.5\%) patients versus 47 of $78(60.3 \%)$ control subjects had 11 to 50 nevi, 12 of 78 (15.4\%) patients versus 14 of 78 (17.9\%) control subjects had 51 to 100 nevi, and 10 of $78(12.8 \%)$ patients versus 4 of $78(5.1 \%)$ control subjects had more than 100 nevi.

In melanoma patients, common nevi were more prevalent on the legs than in control patients (13 vs. 7). The common nevi count didn't differ for other sites between melanoma patients and controls. For Dysplastic nevi, they were most often located on trunk for both melanoma patients and controls, but the nevi count on trunk, legs didn't differ statistically between the groups (P:0.420) (P:0.114).

Logistic regression analysis, showed no specific and statistical significant feature for melanoma (Table 2).
Table 1: Clinical features of patients and controls

\begin{tabular}{lccc}
\hline & Patients & Controls & P value \\
\hline Age & $55.50 \pm 12.91$ & $49.30 \pm 15.21$ & 0.062 \\
Common nevi & $40.96 \pm 37.81$ & $36.66 \pm 28.56$ & 0.260 \\
Head and neck & $9.62 \pm 8.37$ & $7.25 \pm 5.29$ & 0.115 \\
Upper extremities & $8.25 \pm 8.24$ & $10.27 \pm 9.60$ & 0.074 \\
Trunk & $9.73 \pm 9.31$ & $11.94 \pm 9.28$ & 0.084 \\
Lower extremities & $13.36 \pm 11.89$ & $7.2 \pm 4.39$ & 0.035 \\
Clark nevi & $3.37 \pm 4.77$ & $1.85 \pm 1.68$ & 0.382 \\
Head and neck & $0.64 \pm 1.72$ & $0.36 \pm 0.62$ & 0.075 \\
Upper extremities & $0.45 \pm 1.54$ & $0.11 \pm 0.71$ & 0.093 \\
Trunk & $2.08 \pm 1.10$ & $1.01 \pm 0.13$ & 0.420 \\
Lower extremities & $0.19 \pm 0.41$ & $0.37 \pm 0.22$ & 0.114 \\
\hline
\end{tabular}

Table 2: Risk factors for Melanoma

\begin{tabular}{lccc}
\hline & P value & Odds ratio & $\begin{array}{c}\text { 95\% confidence } \\
\text { interval }\end{array}$ \\
\hline Age & 0.53 & 0.962 & $0.938-0.987$ \\
Gender & 0.806 & 0.917 & $0.461-1.826$ \\
Overall nevi count & 0.376 & 1.006 & $0.993-1.018$ \\
Clark nevi count & 0.054 & 0.799 & $0.643-0.994$ \\
\hline
\end{tabular}

\section{DISCUSSION}

Dysplastic nevus (DN) was defined as a clinical and dermatopathological described lesion in melanomaprone families. B-K mole syndrome was defined as in 15 patients with melanoma, as having $<10$ to $>100$ nevi, with various sizes (from $5-15 \mathrm{~mm}$ in diameter), irregular border, and multiple colors in Dysplastic et al's report [1]. On the other side, in their report two individuals developed melanoma without having atypical nevus.

The presence of a large number of nevi (>50) with several, atypical clinical features was accepted as a criteria of Familial Atypical Multiple Mole Melanoma (FAMMM) syndrome or Dysplastic Nevus Syndrome (DNS) in 1992 National Institutes of Health Consensus [3]. Having more than 100 nevi with a diameter of $2 \mathrm{~mm}$ or larger; more than 50 nevi if the patient is younger than 20 years of age were scored as one point according to the British group dysplastic nevus syndrome score [8].

The Dutch Working Group, described the atypical nevus as being $>5 \mathrm{~mm}$ in size, or having a vague border, asymmetric shape, irregular pigmentation, and red hue [9].

Harada, Ackerman and Kittler were the first to indicate the importance of congenital melanocytic nevus $(\mathrm{CMN})$, and proposed that these $\mathrm{DN}$ are actually $\mathrm{CMN}$, in $2007[10,11]$. It is accepted now, 'dysplastic 
nevus,' cannot be defined by clinical or dermoscopic examination and therefore it is a histological term. Therefore, we preferred to use the term 'Dysplastic nevus' instead of 'Dysplastic Nevus' in this study.

While these hypotheses are still debated, we aimed to investigate the association between nevus count (both dysplastic and overall) and melanoma development. There is sufficient clinical evidence indicating that melanoma most commonly develops de novo, infrequently melanoma arises from preexisting nevi, which may be either common nevus or Dysplastic nevus. According to the literature, it appears that $20 \%$ to $30 \%$ of melanomas appear to arise from nevi [12].

Based on current data, there is evidence that a high nevus count correlates with a higher risk of melanoma [6]. In our study of Turkish population, we didn't find an elevated melanoma risk in patients with high nevus counts. Our results are in contrast with the results of several casecontrol studies showing that high total-body nevus counts are the major risk factor for the development of MM [6,7]. This result may be due to genetic factors, Fitzpatrick skin type of Turkish population, and increased sun exposure resulting from the geographic region of our country. The populations in other studies mostly had Fitzpatrick skin type 1, 2 or 3, whereas in our country, Fitzpatrick skin type 4 and 5 are common in general population. The habit of sunscreen usage are lower than the individuals from Europe, the USA and Australia.

Two genetic variants, at 9p21 and 22q13, have recently been identified by genome-wide association studies (GWAS) to be associated with melanocytic nevi development [13]. A recent GWAS identified a novel susceptibility locus known as nidogen 1 (NIDl) on lq42 for nevus count and melanoma risk [14]. Results of the study indicated that increased expression of nidogen in one variant NIDl SNP (rs10754833 T allele) was significantly associated with decreased melanoma risk (OR, 0.86). Since these studies were carried out in Europe and USA, these genetic mutations were not identified in Asian patients. We propose that the similarity of nevus count between patients and controls originate from genetic alterations of different geographic regions.

In conclusion, our data show that (1) high counts of common nevus on the legs in melanoma patients; (2) no difference for number of common nevus; (3) no difference for number of Dysplastic nevi; (4) similar risk of melanoma for individuals with few or more moles. All the patients require a careful screening for melanoma.

\section{REFERENCES}

1. Clark WH Jr, Reimer RR, Greene M, Ainsworth AM, Mastrangelo MJ. Origin of familial malignant melanomas from heritable melanocytic lesions. 'The B-K mole syndrome'. Arch Dermatol. 1978;114:732-8.

2. Lynch HT, Frichot BC III, Lynch JF. Familial atypical multiple mole-melanoma syndrome. J Med Genet. 1978;15:352-6.

3. NIH Consensus conference. Diagnosis and treatment of early melanoma. JAMA 1992;268:1314-9.

4. Swerdlow AJ, English J, MacKie RM, O’Doherty CJ, Hunter JA, Clark J, et al. Benign melanocytic nevi as a risk factor for malignant melanoma. Br Med J (Clin Res Ed). 1986;292:1555-9.

5. Weinstock MA. Cutaneous melanoma. J Am Acad Dermatol. 1993;28:666-8.

6. Rieger E, Soyer HP, Garbe C, Büttner P, Kofler R, Weiss J, et al. Overall and site-specific risk of malignant melanoma associated with nevus counts at different body sites: a multicenter case-control study of the German Central Malignant-Melanoma Registry. Int J Cancer. 1995;62:393-7.

7. MacKie RM, Freudenberger T, Aitchison TC. Personal risk-factor chart for cutaneous melanoma. Lancet. 1989;2:487-90.

8. Bishop JA, Wachsmuth RC, Harland M, Bataille V, Pinney E, Mack P, et al. Genotype/phenotype and penetrance studies in melanoma families with germline CDKN2A mutations. J Invest Dermatol. 2000;114:28-33.

9. Bergman W, van Voorst Vader PC, Ruiter DJ. [Dysplastic nevi and the risk of melanoma: a guideline for patient care]. Ned Tijdschr Geneeskd. 1997;141:2010-4.

10. Harada K, Ackerman AB. Less than $10 \%$ of primary cutaneous melanomas, worldwide, are associated with a melanocytic nevus and that nevus usually is congenital, not "dysplastic". Dermatopathol Pract Concept. 2005;11:3.

11. Ackerman AB, Kittler H. Lack of unanimity about the character of "dysplastic nevus" and "common acquired nevus" in published photomicrographs and clinical photographs. Dermatopathol Pract Concept. 2007;13:1

12. Marks R, Dorevitch AP, Mason G. Do all melanomas come from "moles"? A study of the histological association between melanocytic nevi and melanoma. Australas J Dermatol. 1990;31:77-80.

13. Falchi M, Bataille V, Hayward NK, Duffy DL, Bishop JA, Pastinen T, et al. Genome-wide association study identifies variants at 9 p21 and 22q13 associated with development of cutaneous nevi. Nat Genet. 2009;41:915-9.

14. Nan H, Xu M, Zhang J, Zhang M, Kraft P, Qureshi AA, et al. Genome-wide association study identifies nidogen 1 (NID1) as a susceptibility locus to cutaneous nevi and melanoma risk. Hum Mol Genet. 2011;20:2673-9.

Copyright by Fatma Pelin Cengiz, et al. This is an open-access article distributed under the terms of the Creative Commons Attribution License, which permits unrestricted use, distribution, and reproduction in any

medium, provided the original author and source are credited.

Source of Support: Nil, Conflict of Interest: None declared. 